\title{
Bisoprolol in idiopathic pulmonary arterial hypertension: an explorative study
}

\author{
Jasmijn S.J.A. van Campen (10 1,7, Karin de Boer ${ }^{2,7}$, Mariëlle C. van de Veerdonk ${ }^{1,2}$, \\ Cathelijne E.E. van der Bruggen ${ }^{1}$, Cor P. Allaart ${ }^{2}$, Pieter G. Raijmakers ${ }^{3}$, \\ Martijn W. Heymans ${ }^{4}$, J. Tim Marcus ${ }^{5}$, Hendrik J. Harms ${ }^{1,3}$, M. Louis Handoko ${ }^{2}$, \\ Frances S. de Man $^{1,6}$, Anton Vonk Noordegraaf ${ }^{1}$ and Harm-Jan Bogaard ${ }^{1,6}$
}

Affiliations: ${ }^{1}$ Dept of pulmonary medicine, Institute for cardiovascular research, VU University medical center, Amsterdam, The Netherlands. ${ }^{2}$ Dept of cardiology, Institute for cardiovascular research, VU University medical center, Amsterdam. The Netherlands. ${ }^{3}$ Dept of nuclear medicine and PET-research, VU University medical center, Amsterdam, The Netherlands. ${ }^{4}$ Dept of epidemiology, VU University medical center, Amsterdam, The Netherlands. ${ }^{5}$ Dept of physics and medical technology, VU University medical center, Amsterdam, The Netherlands. ${ }^{6}$ Dept of physiology, Institute for cardiovascular research, VU University medical center, Amsterdam, The Netherlands. ${ }^{7}$ Both authors contributed equally.

Correspondence: Harm-Jan Bogaard, Dept of pulmonary medicine, VU University Medical Center, ZKH 4f.010, De Boelelaan 1117, 1081 HV Amsterdam, The Netherlands. E-mail: hj.bogaardavumc.nl

ABSTRACT While beta-blockers are considered contraindicated in pulmonary arterial hypertension $(\mathrm{PAH})$, the prognostic significance of sympathetic nervous system over-activity suggests a potential benefit of beta-blocker therapy. The aim of this randomised, placebo-controlled, crossover, single centre study was to determine the effects of bisoprolol on right ventricular ejection fraction (RVEF) in idiopathic PAH (iPAH) patients. Additional efficacy and safety parameters were explored.

Patients with optimally treated, stable iPAH (New York Heart Association functional class II/III) were randomised to placebo or bisoprolol. Imaging and functional measurements were performed at baseline, crossover and end of study.

$18 \mathrm{iPAH}$ patients were included, because inclusion faltered before enrolment of the targeted 25 patients. 17 patients completed 6 months of bisoprolol, 15 tolerated bisoprolol, one patient required intravenous diuretics. Bisoprolol was associated with a lower heart rate ( 17 beats per minute, $\mathrm{p}=0.0001$ ) but RVEF remained unchanged. A drop in cardiac index $\left(0.5 \mathrm{~L} \cdot \mathrm{min}^{-1} \cdot \mathrm{m}^{-2}, \mathrm{p}=0.015\right)$ was observed, along with a trend towards a decreased 6-min walking distance (6MWD).

Although careful up-titration of bisoprolol was tolerated by most patients and resulted in a decreased heart rate, no benefit of bisoprolol in iPAH was demonstrated. Decreases in cardiac index and 6MWD suggest a deteriorated cardiac function. The results do not favour the use of bisoprolol in iPAH patients.

@ERSpublications

A bisoprolol dose that significantly reduced heart rate was not associated with a significant change in RVEF in PAH http://ow.ly/j5D0300OgGz

Editorial comment in Eur Respir J 2016; 48: 617-618

This article has supplementary material available from erj.ersjournals.com

Received: Sept 212015 | Accepted after revision: May 212016 | First published online: July 72016

Clinical trial: The study was registered at clinicaltrials.gov with identifier number NCT01246037 and clinicaltrialsregister.eu (EudraCT) with identifier number 2010-020424-21.

Support statement: This research was financially supported by a ZonMW grant (number: 95110079). Funding information for this article has been deposited with the Open Funder Registry.

Conflict of interest: Disclosures can be found alongside this article at erj.ersjournals.com

Copyright OERS 2016 


\section{Introduction}

Idiopathic pulmonary arterial hypertension (iPAH) is a rare disease with a prevalence of 5-15 per million adults $[1,2]$. iPAH is characterised by progressive pulmonary vascular remodelling and the associated increase in right ventricular (RV) afterload eventually leads to right heart failure and premature death. Even with the best available treatment, prognosis remains poor [3]. Current medical treatments aim to dilate small pulmonary arteries, but recent data suggest that, even if treatment reduces RV afterload, RV function in many iPAH patients continues to deteriorate, which then predicts a poor outcome [4]. Therefore, directly targeted RV therapy may be of additional benefit.

Despite the importance of beta-blockers in left heart failure management, current guidelines advise against their use in pulmonary arterial hypertension (PAH) patients $[5,6]$. This recommendation is based on the premise that the immediate negative inotropic and chronotropic effects of beta-blockers will result in systemic hypotension and a decreased exercise capacity and is supported by several publications [7-9].

However, the sympathetic nerve system (SNS) is excessively active in PAH patients, a phenomenon associated with increased mortality [10] and suggesting a benefit of beta-blocker treatment [11-13]. Preclinical studies demonstrated that beta-blockers improve RV function in experimental pulmonary hypertension in an afterload-independent way [14-17]. Furthermore, PERROs et al. [17] recently provided in vitro and preclinical evidence that beta-blocker treatment may not only improve RV function directly, but also indirectly through improvement of endothelial dysfunction and reversal of pulmonary vascular remodelling.

The key question that arises is whether positive effects on myocardial remodelling and oxygen efficiency can negate any acute negative inotropic and chronotropic effects of chronic beta-blocker treatment in $\mathrm{PAH}$. The aim of this randomised, placebo-controlled, crossover, single centre study was to determine the effects of bisoprolol treatment on right ventricular ejection fraction (RVEF) in iPAH patients. A number of additional efficacy and safety parameters were explored.

\section{Methods}

This was a proof-of-concept single centre study with a 12-month prospective, randomised, double-blind, placebo-controlled, crossover design and a half year open-label extension trial. The study was registered at clinicaltrials.gov (NCT01246037) and EudraCT (2010-020424-21). The study was initially designed to detect a $3 \%$ difference in RVEF between placebo and bisoprolol, but failed to enrol the targeted 25 study patients.

\section{Patients}

All iPAH patients older than 18 years in New York Heart Association (NYHA) functional class II and III were screened. Eligible patients were in sinus rhythm and clinically stable on PAH specific medication. Inclusion and exclusion criteria are described in the supplementary material.

\section{Study protocol}

After extensive baseline assessments (described later) patients were randomised 1:1 to either bisoprolol or placebo. Treatment was started at a dosage of $1.25 \mathrm{mg}$ (half a tablet), once daily and was gradually increased to achieve the highest tolerated dosage with a maximum dosage of $10 \mathrm{mg}$ (four tablets). Patients were re-examined every 2 weeks and when possible the dosage was increased by $1.25 \mathrm{mg}$. The dosage was not increased, or was reduced if necessary, in case of a drop in systolic systemic pressure $<90 \mathrm{mmHg}$, progression of heart failure, clinically relevant bradycardia or a heart rate below 60 beats per minute, increased symptoms, or a drop in 6-min walking distance (6MWD) of $>10 \%$. Diuretic dose was guided by clinical examination. On a monthly basis blood was drawn and ECG, quality of life (assessed using the Minnesota living with heart failure questionnaire (MLHFQ)) and 6MWD were determined. 4 months into the study, drug doses were not further increased. Patients were re-admitted 6 months after the start of the study to repeat all baseline assessments. Study medication was tapered down and stopped after 2 weeks. Subsequently, patients were crossed over to the alternative medication and in the following 6 months, the same procedures were repeated (figure 1).

At baseline, before cross over and after 1 year of study, patients were admitted for assessment of outcome parameters using cardiac magnetic resonance imaging (CMRI), right heart catheterisation, exercise testing, blood sampling, heart rate variability measurements, ${ }^{11} \mathrm{C}$-acetate positron emission tomography (PET) scans and quality of life questionnaires as described in the supplementary material, which also provides information on clinical deterioration, medical ethical board approval and serious adverse events. 


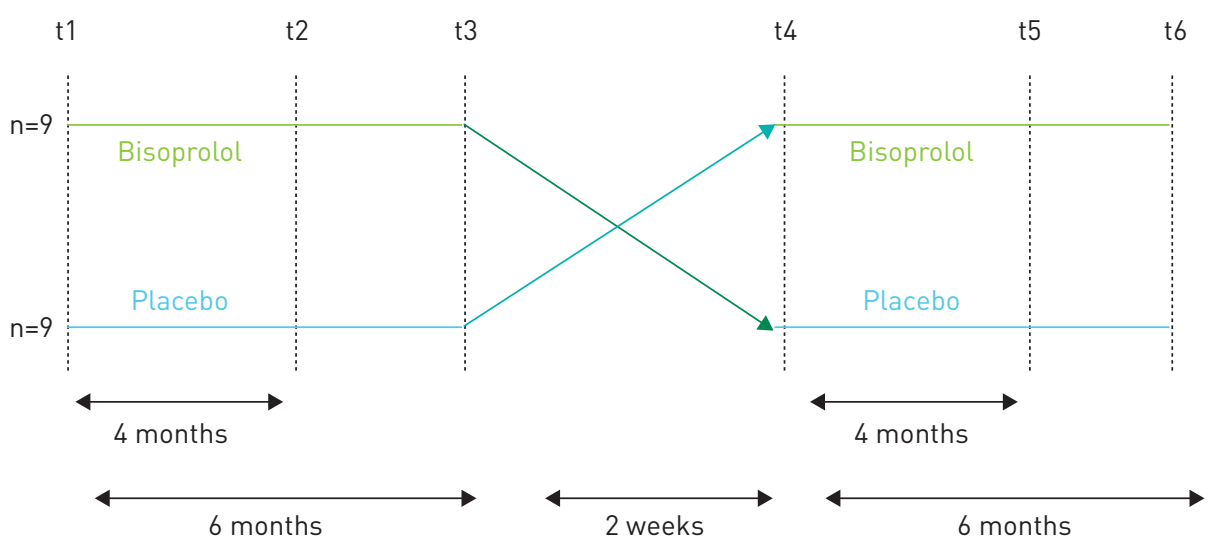

FIGURE 1 Study scheme. Clinically stable idiopathic pulmonary arterial hypertension patients were included in the study. At baseline, after 6 months and at the end of the study $(t=1,3$ and 6$)$ patients were admitted for extensive testing. Between $\mathrm{t} 1-\mathrm{t} 2$ and $\mathrm{t} 4-\mathrm{t} 5$, patients were examined every 2 weeks for up-titration of bisoprolol or placebo. Between $\mathrm{t} 2-\mathrm{t} 3$ and $\mathrm{t} 5 \mathrm{-t} 6$ all patients received a stable drug dose. Medications were tapered and then stopped between $\mathrm{t} 3$ and $\mathrm{t} 4$, and after $\mathrm{t} 6$, unless patients entered the open-label extension study.

\section{Statistical analysis}

Data are presented as mean \pm SD. To compare the effects of 6 months of bisoprolol and placebo treatment a linear mixed model was used to control for the repeated outcome assessments within patients (see the supplementary material).

A $3 \%$ change in RVEF was considered a clinically significant treatment effect $[4,18]$. The power calculation showed that, with $80 \%$ power, 25 patients were required in the crossover study, including the open-label extension trial. Blinding codes were broken on the last day of the third admission or at the moment of early termination of the study. All data were analysed in a blinded fashion and according to an intention-to-treat principle. Statistics were performed using IBM SPSS Statistics 20 (IBM Corp., Armonk, NY, USA) and GraphPad Prism 6 (GraphPad Software, La Jolla, CA, USA).

\section{Results}

\section{Patient inclusion}

Prior to inclusion all patients were treated in our hospital, an academic referral centre for PAH in the Netherlands. 27 out of 45 patients eligible for this study declined because of the high study burden, including frequent hospital visits and invasive testing. 18 patients were enrolled into the study from February 2011 until January 2014 (figure 2). Patient recruitment had become extremely slow at that time, with only one additional patient recruited in the past year of the study. Together with the Institutional Review Board of our institution and the Data Safety Monitoring Board we made the decision that the study should be closed. Failure to enrol the targeted 25 patients was related to stringent eligibility criteria and the high study burden. NYHA classes II and III were equally represented, mean age was 48 years and there was a strong female predominance (one male patient was included). Patient baseline characteristics, dosages of placebo and bisoprolol, and diuretic medication changes are shown in table 1 and per patient in table S1.

In the placebo arm of the study, 16 out of 18 patients received placebo for the full 6 months: one patient was put back on bisoprolol after 2 months because of symptomatic palpitations and one patient accidentally received bisoprolol during part of the placebo half year (improper packaging of study medication at the pharmacy). In the bisoprolol arm of the study, 17 patients received bisoprolol for 6 months: one patient withdrew from the study directly after crossover and before the first dose of bisoprolol because she found the study too burdensome. A complete paired set of study data is available from 16 patients. All available data were included in the analysis.

\section{Adverse events}

Five serious adverse events (SAEs) were reported, one of which was directly related to the study medication. This patient had to be admitted for treatment of fluid retention with intravenous diuretics after she started with bisoprolol. One SAE concerned the described pharmacy error. Three other SAEs were related to bacterial infections, which were not obviously related to the study drug or protocol.

\section{Tolerability}

17 patients completed the 6-month bisoprolol treatment arm, but two (12\%) of these patients received a final dose of 0 tablets because of systemic hypotension or a $10 \%$ drop in exercise capacity. Both patients 


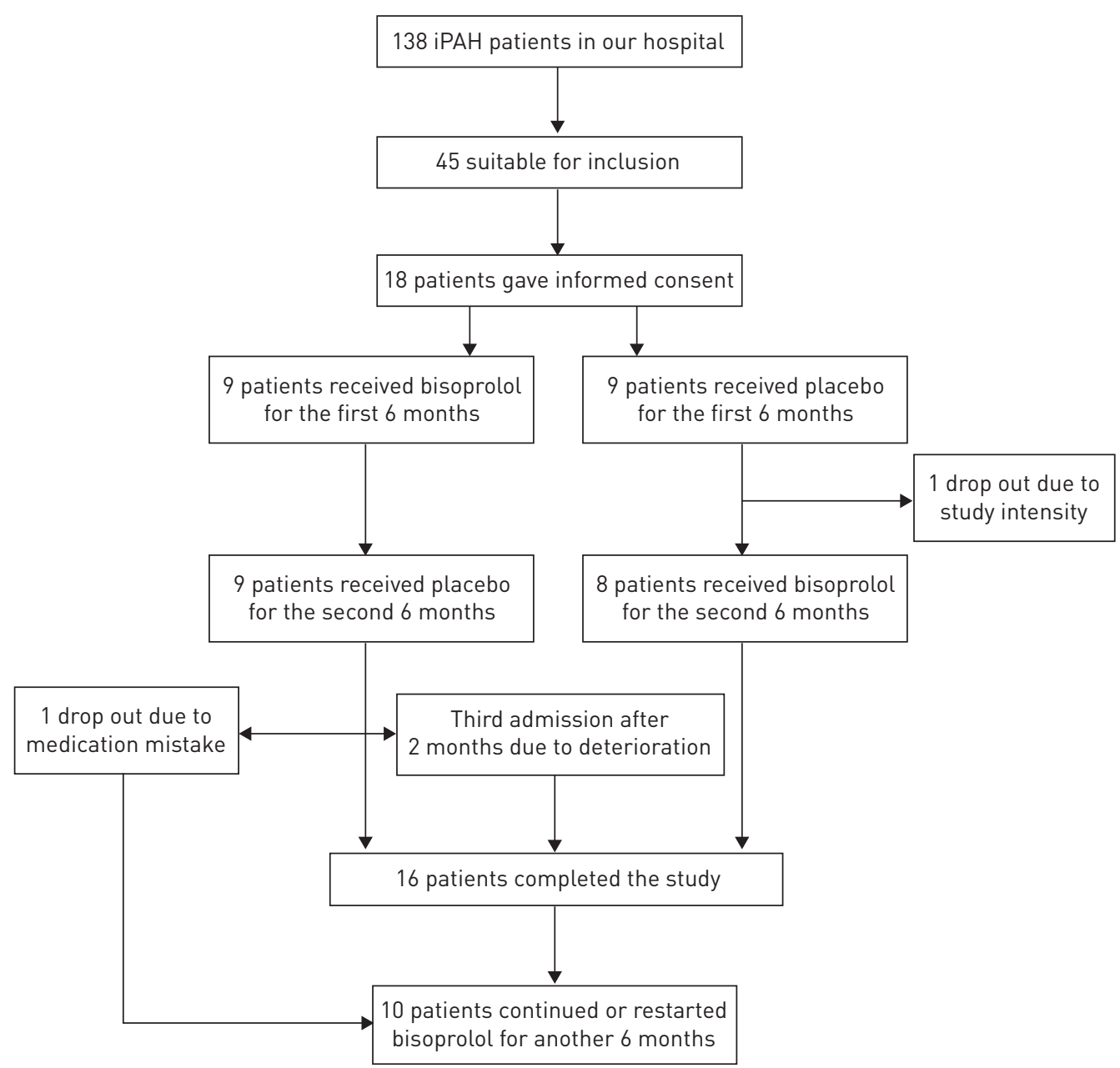

FIGURE 2 Flowchart of inclusion of idiopathic pulmonary arterial hypertension (iPAH) patients in the study.

stayed enrolled in the study and the data acquired after 6 months in the bisoprolol study arm was fully analysed (intention-to-treat analysis). Full up-titration to a maximal dose of four tablets was impossible in 12 placebo treated patients because of a low baseline systemic blood pressure. One patient stopped the placebo tablets according to protocol for this reason. 11 (65\%) out of the 17 patients continued or restarted bisoprolol after the end of the study following consultation with their treating clinician. One patient stopped after 3 months in the open-label extension study because of erectile dysfunction. The other ten patients completed another half year of bisoprolol. These data are included in the analysis.

\section{Signs of congestive heart failure and other side-effects}

Worsening of fluid retention is a well-known side-effect of beta-blocker treatment in congestive heart failure. As described, one patient was admitted for i.v. diuretics after the first weeks of bisoprolol. After her admission, she was stable on oral diuretics and continued $1.25 \mathrm{mg}$ of bisoprolol for half a year. It was not possible to increase the dosage, because of hypotension. On average, however, body weight, N-terminal pro-brain natriuretic peptide (NT-proBNP), serum sodium and total doses of loop diuretics were not significantly affected by bisoprolol treatment (table 1 and per patient in table S1). Some patients complained about tiredness or a subjective feeling of a drop in exercise tolerance. However, this was not clearly reflected in the quality of life questionnaire or a drop of $>10 \%$ in $6 \mathrm{MWD}$ and patients with placebo had similar complaints. One patient described temporary feelings of depression while treated with bisoprolol.

\section{Systemic haemodynamics}

Systolic blood pressure remained stable after bisoprolol treatment. A slight reduction in diastolic blood pressure (decrease of $6 \mathrm{mmHg}, \mathrm{p}=0.024$ ) did not result in a significant change in mean systemic pressure $(-2.7 \mathrm{mmHg}, \mathrm{p}=0.32)$ (table 1$)$. 
TABLE 1 Baseline characteristics and results after 6 months of placebo and after 6 months of bisoprolol

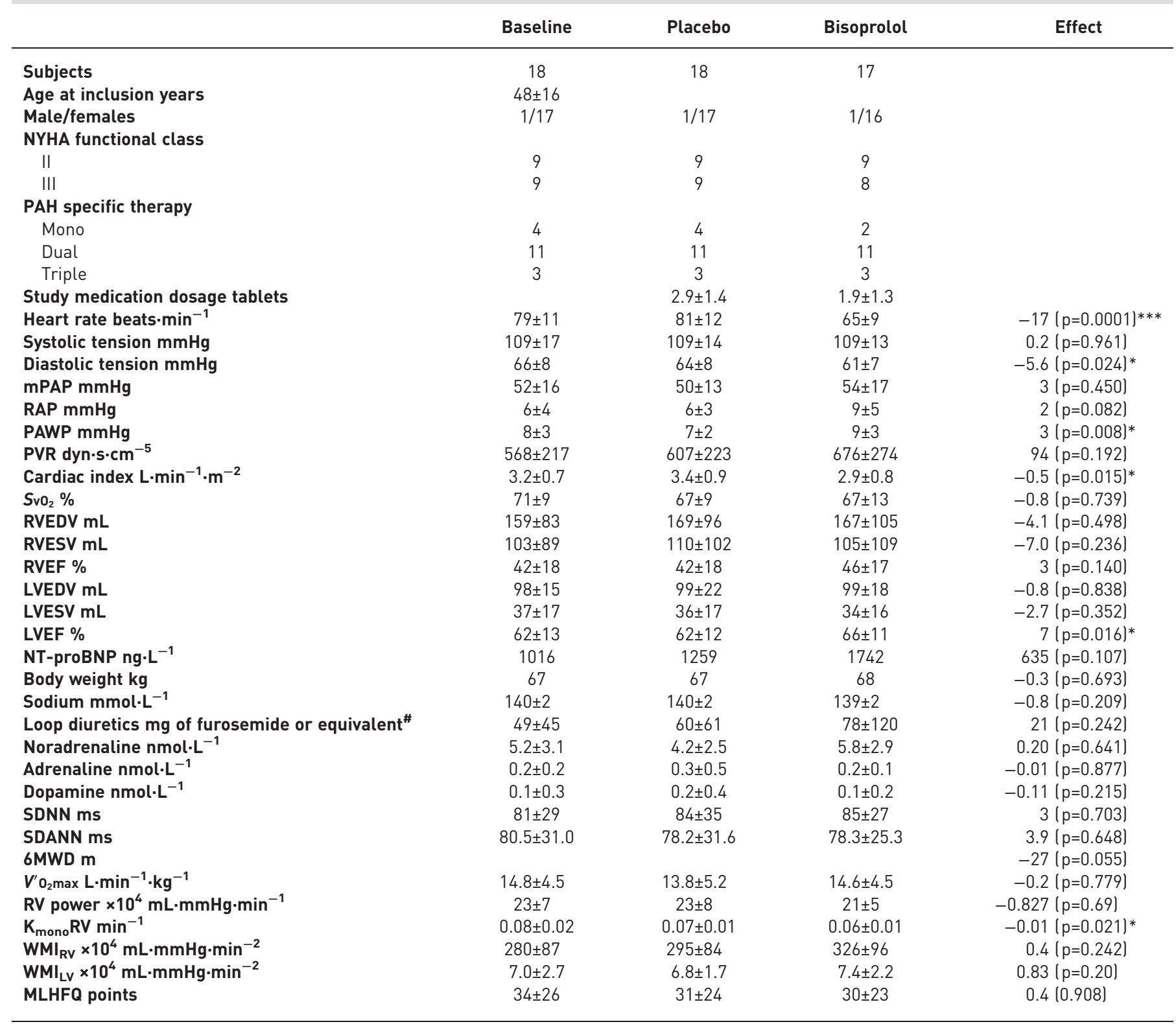

Data are presented as $n$, mean \pm SD or the effect of 6 months of bisoprolol treatment compared with 6 months of placebo treatment calculated with a linear mixed model and corrected for dosage of study medication. NYHA: New York Heart Association; PAH: pulmonary arterial hypertension; mPAP: mean pulmonary arterial pressure; RAP: right atrial pressure; PAWP: pulmonary artery wedge pressure; PVR: pulmonary vascular resistance; $\mathrm{SvO}_{2}$ : mixed venous oxygen saturation; RVEDV: right ventricular end diastolic volume; RVESV: right ventricular end systolic volume; RVEF: right ventricular ejection fraction; LVEDV: left ventricular end diastolic volume; LVESV: left ventricular end systolic volume; LVEF: left ventricular ejection fraction; NT-proBNP: N-terminal pro-brain natriuretic peptide; SDNN: standard deviation of all normal RR intervals in a 24-h ECG; SDANN: standard deviation of the average normal RR intervals for all 288 5-min segments of a 24-h ECG recording; 6MWD: 6-min walking distance; $V^{\prime} \mathrm{O}_{2}$ max: maximal oxygen uptake per $\mathrm{kg}$; RV: right ventricular; RV power: right ventricular power output; $\mathrm{K}_{\text {mono }} \mathrm{RV}$ : rate of ${ }^{11} \mathrm{C}$-acetate clearance determined by mono-exponential curve fitting; WMI: work myocardial index; LV: left ventricular; MLHFQ: Minnesota living with heart failure questionnaire. ${ }^{\#}: 1 \mathrm{mg}$ bumetanide is calculated as 40 mg furosemide. ${ }^{*}: \mathrm{p}<0.05 ;{ }^{* * *}: \mathrm{p}<0.001$.

\section{Sympathetic activity}

Sympathetic activity is reflected by inter alia heart rate. Overall, bisoprolol-treatment was associated with a reduction in heart rate of 17 beats per minute $(\mathrm{p}=0.0001)$ (figure 3 ). None of the other heart rate variability (HRV) measurements showed a significant change after beta-blocker therapy (table 1 and table S2). No significant effects of bisoprolol were found on plasma concentrations of noradrenaline, adrenaline or dopamine (table 1). 

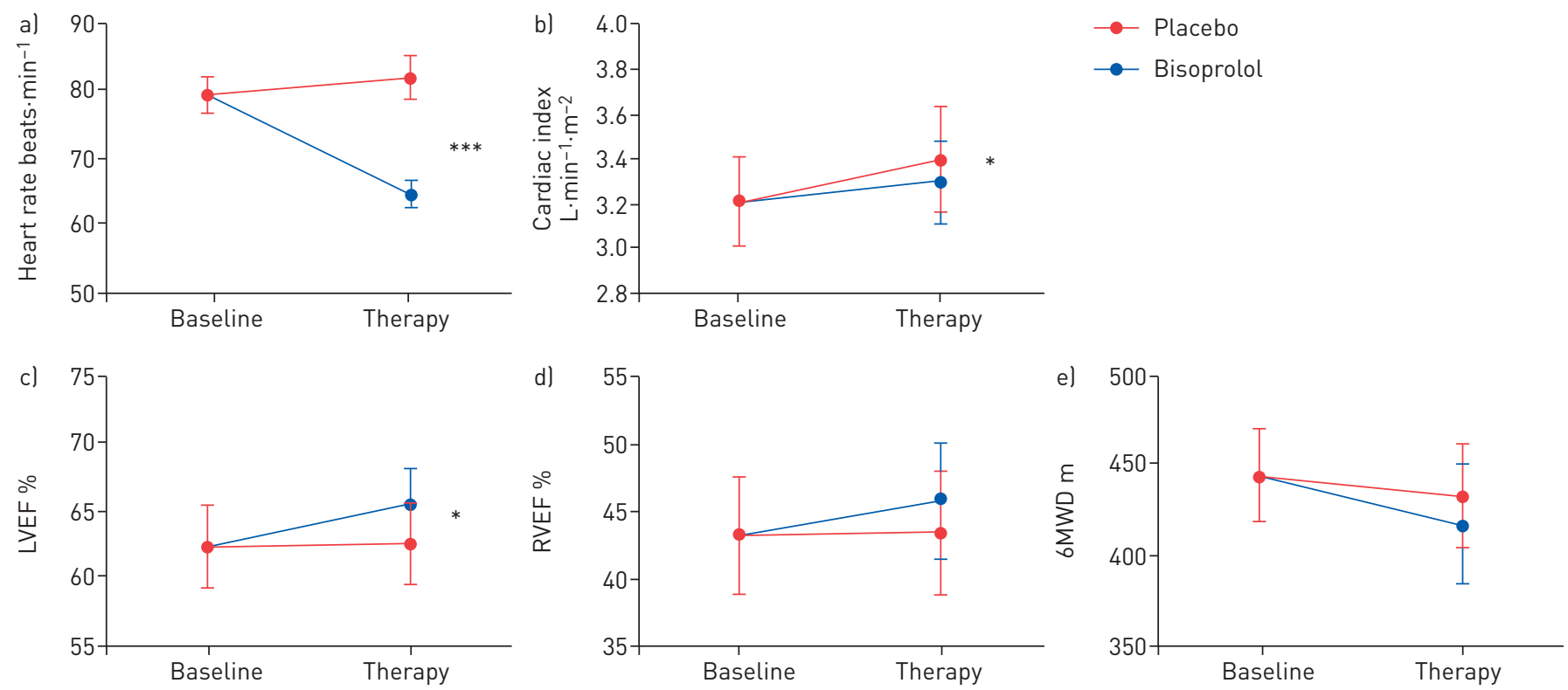

FIGURE 3 Effect on haemodynamics and 6-min walking distance (6MWD) of 6 months of bisoprolol treatment compared with the effect of 6 months of placebo treatment. Effects were calculated using a linear mixed model and corrected for dosage of study medication. a) Heart rate decreased by 17 beats $\cdot \mathrm{min}^{-1}$ after bisoprolol treatment $(\mathrm{p}=0.0001)$. b) Cardiac index decreased by $0.5 \mathrm{~L} \cdot \mathrm{min}^{-1} \cdot \mathrm{m}^{-2}$ after bisoprolol treatment ( $\left.\mathrm{p}=0.015\right)$. c) Left ventricular ejection fraction (LVEF) increased by $7 \%$ after bisoprolol treatment ( $p=0.016$ ). d) Right ventricular ejection fraction (RVEF) increased by $3 \%$ after bisoprolol treatment $(p=0.140)$. e) 6MWD decreased by $27 \mathrm{~m}$ after bisoprolol treatment $(p=0.055) .{ }^{*}: p<0.05 ;{ }^{* * *}: p<0.001$.

\section{Right and left ventricular function}

Bisoprolol treatment was associated with a small but statistically not significant improvement in RVEF (increase 3\%, $\mathrm{p}=0.140$ ) (figure 3), while right ventricular end diastolic volume and right ventricular end systolic volume remained unchanged (table 1). Right ventricular stroke volume was unaltered and given the reduction in heart rate, a significant diminution in resting cardiac index was observed (decrease of $0.5 \mathrm{~L} \cdot \mathrm{min}^{-1} \cdot \mathrm{m}^{-2}, \mathrm{p}=0.015$ ) (figure 3). Mean pulmonary arterial pressure, right atrial pressure and pulmonary vascular resistance remained stable and pulmonary artery wedge pressure (PAWP) showed a modest increase with bisoprolol $(+3 \mathrm{mmHg}, \mathrm{p}=0.008)$ (table 1$)$. While left ventricular end diastolic volume, left ventricular end systolic volume and left ventricular stroke volume remained unchanged with bisoprolol (table 1), left ventricular ejection fraction (LVEF) improved significantly (increase of 7\%, $\mathrm{p}=0.016$ ) (figure 3).

\section{Exercise capacity}

Although there was great variability in the responses between patients, bisoprolol tended to decrease 6MWD (decrease $27 \mathrm{~m}, \mathrm{p}=0.055$ ) (figure 3), while maximal oxygen uptake per $\mathrm{kg}\left(V^{\prime} \mathrm{O}_{2} \max\right.$ ) remained unchanged (decrease of $0.2 \mathrm{~mL} \cdot \mathrm{min}^{-1} \cdot \mathrm{kg}^{-1}, \mathrm{p}=0.779$ ) (table 1).

\section{Myocardial efficiency}

10 patients underwent an ${ }^{11} \mathrm{C}$-acetate scan at baseline, and after crossover and follow-up. In other patients not all scans were performed due to software issues and failed tracer synthesis. RV rate of ${ }^{11} \mathrm{C}$-acetate clearance determined by mono-exponential curve fitting, which reflects RV oxygen consumption [19], was significantly reduced after bisoprolol therapy $\left(-0.01 \mathrm{~min}^{-1}, \mathrm{p}=0.021\right)$. There was no significant effect on RV power output or RV mechanical efficiency (figure 4).

\section{Quality of life}

Health-related quality of life measured using the MLHFQ was not significantly affected by bisoprolol (table 1).

\section{Discussion}

Failure to enrol 25 patients precludes an answer to the main question of whether 6 months of bisoprolol treatment leads to an improvement in RVEF in iPAH. Slow patient recruitment ultimately led to premature closing of the study, and with 18 patients included this study was underpowered to detect an improvement in RVEF. While a significant increase in LVEF was observed after bisoprolol treatment, worrisome safety signals consisted of a significant decrease in cardiac index and a trend towards a decreased 6MWD. 

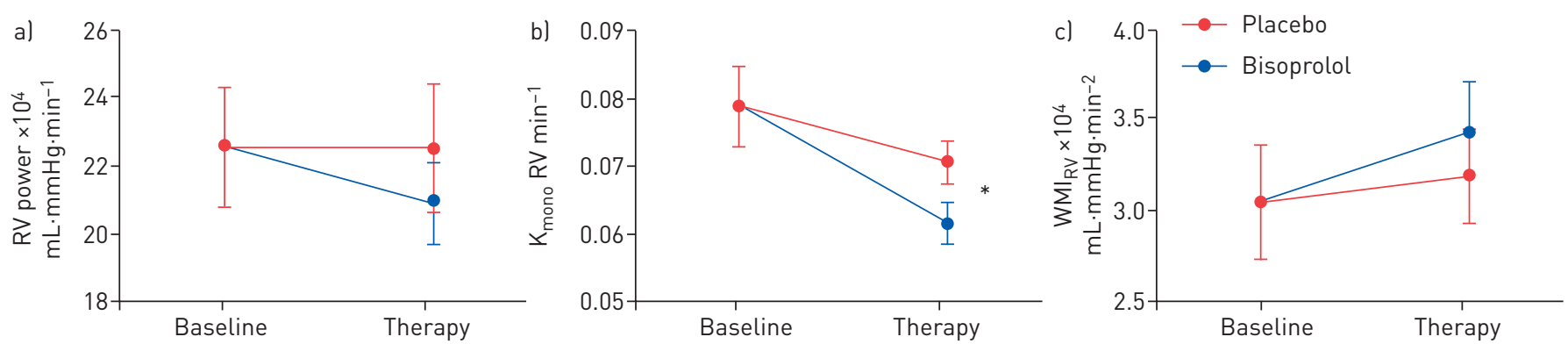

FIGURE 4 Effect on right ventricular power output and efficiency of 6 months of bisoprolol treatment compared with 6 months of placebo treatment Effects were calculated using a linear mixed model and corrected for dosage of study medication. a) Right ventricular power output (RV power) decreased by $0.827 \times 10^{4} \mathrm{~mL} \cdot \mathrm{mmHg} \cdot \mathrm{min}^{-1}$ after bisoprolol treatment $(p=0.69)$. b) Rate of ${ }^{11} \mathrm{C}$-acetate clearance determined by mono-exponential curve fitting ( $\mathrm{K}_{\text {mono }} \mathrm{RV}$ ) decreased by $0.012 \mathrm{~min}^{-1}$ after bisoprolol treatment $(\mathrm{p}=0.021)$. c) Work myocardial index of the right ventricle (WMI $\mathrm{RV}$ ) increased by $0.4 \times 10^{4} \mathrm{ml} \cdot \mathrm{mmHg} \cdot \mathrm{min}^{-2}$ after bisoprolol treatment $(\mathrm{p}=0.24){ }^{*}: \mathrm{p}<0.05$.

The tolerability of bisoprolol in this study of iPAH patients seems comparable with what has been reported in left heart failure: 15 (88\%) out of 17 patients tolerated bisoprolol treatment (versus $85 \%$ in left ventricular failure) [6]. 10 (59\%) out of 17 patients decided to continue in the open-label extension trial. One patient had to be admitted for treatment with i.v. diuretics after starting bisoprolol. Overall bisoprolol treatment did not result in an increase in NT-proBNP, fluid retention or diuretic use, and there was no change in systemic blood pressure.

Remarkably, a significant improvement in LVEF was observed. Although this finding was unexpected and unexplained, it raises the possibility that changes in RVEF were to some degree indirect and perhaps related to ventricular interdependence. Also the prolonged filling time can be supposed to contribute to this increase, but no correlation between heart rate and LVEF at baseline, or change in heart rate and change in LVEF was found ( $r=0.26, p=0.29$; and $r=0.11, p=0.59$, respectively).

Along with the improvement in LVEF, a small increase in PAWP was noted. Although there is no certain explanation for this finding, we can speculate that filling pressures were increased due to a longer heart period and some degree of fluid retention. The small increase in PAWP was not associated with the occurrence of pulmonary oedema.

\section{Comparison with earlier studies}

This is the first placebo-controlled study of beta-blocker therapy in patients with iPAH. We used the selective beta-blocker bisoprolol in clinically stable iPAH patients, which is a different approach from those of previous studies. Provencher et al. [7] described the beneficial effects of the withdrawal of the unselective beta-blocker propranolol in patients with portopulmonary hypertension and PEACOCK and Ross [8] reported deleterious effects of beta-blocker therapy in an iPAH patient with acute heart failure. BANDYOPADHYAY et al. [9] observed no positive effects of beta-blocker therapy on survival time or time to clinical worsening in PAH patients, but a tendency towards a shorter walking distance. Despite these reports and based on the experimental and clinical effectiveness of inhibiting sympathetic over-activity in left ventricular failure, several animal studies were preformed to examine potential positive effects of beta-blocker therapy in experimental pulmonary hypertension [14-17]. The degree of heart rate reduction that was achieved in these animal studies was also reached in our clinical study. But the positive effects of beta-blockers on the function and structure of the right ventricle were not reproduced in this clinical study.

Despite the recommendation against beta-blocker use in right heart failure, recent data suggests that $\sim 28 \%$ of Canadian pulmonary hypertension patients are treated with beta-blockers without major complications [20]. We calculated that during the ARIES 1 and 2 studies [21], the sildenafil dose finding study [22] and the sildenafil registration study [23] 14\%, $12 \%$ and 6\%, respectively, of $\mathrm{PAH}$ patients were treated with beta-blockers (unpublished data; courtesy of GlaxoSmithKline and Pfizer), often for reasons related to palpitations. Thenappan et al. [24] and MoretTi et al. [25] showed that beta-blocker therapy is not associated with adverse outcomes in patients with $\mathrm{PAH}$. The recently published pilot study of carvedilol treatment in six PAH patients showed a significant improvement in RVEF and no negative effects on exercise capacity [26].

A small number of almost exclusively female iPAH patients were included in this single centre study, which contrasts sharply with the large beta-blocker trials in left ventricular failure in which thousands of predominantly male patients were included. The effects of bisoprolol on all-cause mortality and cardiovascular death were better in female left ventricular failure patients compared with the effects of other selective beta-blockers, where women had higher plasma concentrations and more side-effects were found $[27,28]$. 
The selective beta-blockers bisoprolol, carvedilol and metoprolol are approved for the treatment of left ventricular failure [6] and have superior survival effects when compared with unselective beta-blockers such as propranolol. We chose to use bisoprolol in this study, a drug with a much higher affinity for the

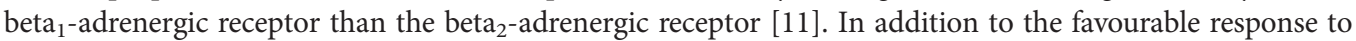
bisoprolol of female left heart failure patients, we expected less of a decline in exercise performance when using a beta ${ }_{1}$-selective agent, because beta $_{2}$ - blockade tends to blunt the exercise-induced increase in skeletal muscle blood flow [11]. Furthermore, bisoprolol causes less peripheral vasodilatation and thereby less systemic hypotension than the beta ${ }^{-}$plus alpha $a_{1}$-adrenergic receptor blocker carvedilol.

\section{Strengths and weaknesses}

The placebo-controlled, crossover design, with an open-label extension in which patients served as their own controls, allowed measurement of effects in a relatively small number of patients. The linear mixed model used for the statistical analyses takes into account the dosage of study medication and thereby accurately assesses treatment effects in a crossover study. Frequent study visits secured the safety of patients but also contributed to the high burden of this study and thereby stagnant inclusion. We were unable to recruit the number of patients that were required according to the power calculation. Therefore this study lacked statistical power to detect a significant change in RVEF, which we chose as the primary outcome parameter. The ideal end-point for this study would have been survival, but due to limited patient numbers this end-point was not feasible and a crossover design was needed. Because we previously showed that a change in RVEF upon treatment is a strong prognostic marker in $\mathrm{PAH}$ [4] and also because the 6MWD has recently been questioned as a surrogate end-point, we chose an increase in RVEF of 3\% as the primary outcome of the study. The possibility that RVEF and LVEF were affected by bisoprolol because of a slowing heart rate and prolonged filling time, seems unlikely as we found no correlation between changes in heart rate and RVEF $(\mathrm{r}=-0.117, \mathrm{p}=0.570)$. However, the concomitant reduction of cardiac output suggests that the increases in RVEF and LVEF cannot be attributed to an improved global cardiac function.

Patient characteristics that may have affected outcome were long-term clinical stability before inclusion and the inclusion of only one male patient. In addition, 14 out of 17 patients in our study used endothelin receptor antagonists, drugs which are known to affect adrenergic signalling [29].

\section{Interpretation and mechanism}

Among the mechanisms contributing to beneficial effects of beta-blocker treatment in left heart failure are a reduction in SNS over-activity, attenuation of cardiac remodelling and hypertrophy, and a reduction of myocardial oxygen consumption [30]. We studied SNS activity through assessment of heart rate variability and catecholamine concentrations in the peripheral blood, but found no significant effects of bisoprolol treatment. There was a near significant effect on the triangular interpolation of NN interval histogram and a trend on the low frequency/high frequency ratio. But, as there is no unique preferred parameter to detect effects on SNS activity in HRV measurements, it is difficult to interpret the data (see the supplementary material) [31-33]. It remains unclear whether the tolerated dosage was sufficient to reduce SNS over-activity, or whether the study was underpowered to address this question.

In left ventricular failure, a high resting heart rate predicts poorer prognosis and reducing the heart rate to 70 beats per minute improves prognosis [34]. Similarly in PAH, a high resting heart rate is associated with a worse prognosis [35]. In this study, the tolerated dosage of bisoprolol resulted in a significant reduction in heart rate and myocardial oxygen consumption. However, in contrast to left heart failure studies [30, 36], bisoprolol did not improve cardiac efficiency.

The deterioration in cardiac index of $0.5 \mathrm{~L} \cdot \mathrm{min}^{-1} \cdot \mathrm{m}^{-2}(\mathrm{p}=0.015)$ in this study was probably responsible for the near significant drop in $6 \mathrm{MWD}$. Although $V^{\prime} \mathrm{O}_{2} \max$ was unaltered, a drop in cardiac index could be seen as a sign of progressive heart failure. One patient had to be admitted for i.v. diuretics after the first month of bisoprolol treatment. On average, however, MLHFQ, body weight, NT-proBNP, serum sodium and total doses of loop diuretics were not significantly affected by bisoprolol treatment and thereby gave no other indication of progressive heart failure.

\section{Clinical relevance}

This underpowered study gives no reason to prescribe bisoprolol in iPAH patients as no positive effects on RVEF were found. The significant drop in cardiac index and near significant decrease in 6MWD, two potentially negative prognostic effects, after bisoprolol treatment were not associated with a worsening quality of life, but rather with a reduced myocardial oxygen consumption. These findings compare with studies of beta-blocker therapy in left heart failure [37]. Further studies are needed to answer the question of whether bisoprolol treatment is associated with serious adverse effects in PAH patients. 


\section{Conclusion}

Although careful up-titration of bisoprolol was tolerated by most patients and resulted in a decreased heart rate, the present study demonstrated no benefit of bisoprolol in iPAH. The drop in cardiac index and reduction in $6 \mathrm{MWD}$ suggest deterioration in cardiac function. This study does not favour the use of bisoprolol in iPAH patients.

\section{Acknowledgements}

Martha Wagenaar, Frank Oosterveer, Herman Groepenhoff and Iris van der Mark of the Pulmonary Department of the VUmc medical center (Amsterdam, the Netherlands) contributed significantly to the planning and execution of the study.

\section{References}

1 Benza RL, Miller DP, Barst RJ, et al. An evaluation of long-term survival from time of diagnosis in pulmonary arterial hypertension from the REVEAL Registry. Chest 2012; 142: 448-456.

2 Humbert M, Sitbon O, Chaouat A, et al. Pulmonary arterial hypertension in France: results from a national registry. Am J Respir Crit Care Med 2006; 173: 1023-1030.

3 Ling Y, Johnson MK, Kiely DG, et al. Changing demographics, epidemiology, and survival of incident pulmonary arterial hypertension: results from the pulmonary hypertension registry of the United Kingdom and Ireland. Am J Respir Crit Care Med 2012; 186: 790-796.

4 van de Veerdonk MC, Kind T, Marcus JT, et al. Progressive right ventricular dysfunction in patients with pulmonary arterial hypertension responding to therapy. J Am Coll Cardiol 2011; 58: 2511-2519.

5 Lau EMT, Tamura Y, McGoon MD, et al. The 2015 ESC/ERS Guidelines for the diagnosis and treatment of pulmonary hypertension: a practical chronicle of progress. Eur Respir J 2015; 46: 879-882.

6 Yancy CW, Jessup M, Bozkurt B, et al. 2013 ACCF/AHA guideline for the management of heart failure: a report of the American College of Cardiology Foundation/American Heart Association Task Force on Practice Guidelines. J Am Coll Cardiol 2013; 62: e147-e239.

7 Provencher S, Herve P, Jais X, et al. Deleterious effects of beta-blockers on exercise capacity and hemodynamics in patients with portopulmonary hypertension. Gastroenterology 2006; 130: 120-126.

8 Peacock A, Ross K. Pulmonary hypertension: a contraindication to the use of $\beta$-adrenoceptor blocking agents. Thorax 2010; 65: 454-455.

9 Bandyopadhyay D, Bajaj NS, Zein J, et al. Outcomes of $\beta$-blocker use in pulmonary arterial hypertension: a propensity-matched analysis. Eur Respir J 2015; 46: 750-760.

10 Ciarka A, Doan V, Velez-Roa S, et al. Prognostic significance of sympathetic nervous system activation in pulmonary arterial hypertension. Am J Respir Crit Care Med 2010; 181: 1269-1275.

11 Triposkiadis F, Karayannis G, Giamouzis G, et al. The sympathetic nervous system in heart failure physiology, pathophysiology, and clinical implications. J Am Coll Cardiol 2009; 54: 1747-1762.

12 Bristow MR, Quaife RA. The adrenergic system in pulmonary arterial hypertension: bench to bedside (2013 Grover Conference series). Pulm Circ 2015; 5: 415-423.

13 Ryan JJ, Archer SL. The right ventricle in pulmonary arterial hypertension: disorders of metabolism, angiogenesis and adrenergic signaling in right ventricular failure. Circ Res 2014; 115: 176-188.

14 Bogaard HJ, Natarajan R, Mizuno S, et al. Adrenergic receptor blockade reverses right heart remodeling and dysfunction in pulmonary hypertensive rats. Am J Respir Crit Care Med 2010; 182: 652-660.

15 de Man FS, Handoko ML, van Ballegoij JJM, et al. Bisoprolol delays progression towards right heart failure in experimental pulmonary hypertension. Circ Heart Fail 2012; 5: 97-105.

16 Drake JI, Gomez-Arroyo J, Dumur CI, et al. Chronic carvedilol treatment partially reverses the right ventricular failure transcriptional profile in experimental pulmonary hypertension. Physiol Genomics 2013; 45: 449-461.

17 Perros F, Ranchoux B, Izikki M, et al. Nebivolol for improving endothelial dysfunction, pulmonary vascular remodeling, and right heart function in pulmonary hypertension. J Am Coll Cardiol 2015; 65: 668-680.

18 Bradlow WM, Hughes ML, Keenan NG, et al. Measuring the heart in pulmonary arterial hypertension (PAH): implications for trial study size. J Magn Reson Imaging 2010; 31: 117-124.

19 Wong YY, Raijmakers P, van Campen J, et al. 11C-Acetate clearance as an index of oxygen consumption of the right myocardium in idiopathic pulmonary arterial hypertension: a validation study using $15 \mathrm{O}$-labeled tracers and PET. J Nucl Med 2013; 54: 1258-1262.

20 So PP-S, Davies RA, Chandy G, et al. Usefulness of beta-blocker therapy and outcomes in patients with pulmonary arterial hypertension. Am J Cardiol 2012; 109: 1504-1509.

21 Galiè N, Olschewski H, Oudiz RJ, et al. Ambrisentan for the treatment of pulmonary arterial hypertension: results of the ambrisentan in pulmonary arterial hypertension, randomized, double-blind, placebo-controlled, multicenter, efficacy (ARIES) study 1 and 2. Circulation 2008; 117: 3010-3019.

22 Galiè N, Ghofrani HA, Torbicki A, et al. Sildenafil citrate therapy for pulmonary arterial hypertension. $N$ Engl J Med 2005; 353: 2148-2157.

23 Simonneau G, Rubin LJ, Galiè N, et al. Addition of sildenafil to long-term intravenous epoprostenol therapy in patients with pulmonary arterial hypertension: a randomized trial. Ann Intern Med 2008; 149: 521-530.

24 Thenappan T, Roy SS, Duval S, et al. $\beta$-blocker therapy is not associated with adverse outcomes in patients with pulmonary arterial hypertension: a propensity score analysis. Circ Heart Fail 2014; 7: 903-910.

25 Moretti C, Grosso Marra W, D'Ascenzo F, et al. Beta blocker for patients with pulmonary arterial hypertension: a single center experience. Int J Cardiol 2015; 184: 528-532.

26 Grinnan D, Bogaard HJ, Grizzard J, et al. Treatment of group I pulmonary arterial hypertension with carvedilol is safe. Am J Respir Crit Care Med 2014; 189: 1562-1564.

27 Simon T, Mary-Krause M, Funck-Brentano C, et al. Sex differences in the prognosis of congestive heart failure: results from the Cardiac Insufficiency Bisoprolol Study (CIBIS II). Circulation 2001; 103: 375-380.

28 Seeland U, Regitz-Zagrosek V. Sex and gender differences in cardiovascular drug therapy. Handb Exp Pharmacol 2012; 214: 211-236. 
29 Lehmann LH, Rostosky JS, Buss SJ, et al. Essential role of sympathetic endothelin A receptors for adverse cardiac remodeling. Proc Natl Acad Sci USA 2014; 111: 13499-13504.

30 Beanlands RS, Nahmias C, Gordon E, et al. The effects of $\beta_{1}$-blockade on oxidative metabolism and the metabolic cost of ventricular work in patients with left ventricular dysfunction: a double-blind, placebo-controlled, positron-emission tomography study. Circulation 2000; 102: 2070-2075.

31 Zhang Y, de Peuter OR, Kamphuisen PW, et al. Search for HRV-parameters that detect a sympathetic shift in heart failure patients on $\beta$-blocker treatment. Front Physiol 2013; 4: 81.

32 Fauchier L, Babuty D, Melin A, et al. Heart rate variability in severe right or left heart failure: the role of pulmonary hypertension and resistances. Eur J Heart Fail 2004; 6: 181-185.

33 Xhyheri B, Manfrini O, Mazzolini M, et al. Heart rate variability today. Prog Cardiovasc Dis 2012; 55: 321-331.

34 Kubon C, Mistry NB, Grundvold I, et al. The role of beta-blockers in the treatment of chronic heart failure. Trends Pharmacol Sci 2011; 32: 206-212.

35 Swiston JR, Johnson SR, Granton JT. Factors that prognosticate mortality in idiopathic pulmonary arterial hypertension: a systematic review of the literature. Respir Med 2010; 104: 1588-1607.

36 Bengel FM, Ueberfuhr P, Schiepel N, et al. Myocardial efficiency and sympathetic reinnervation after orthotopic heart transplantation: a noninvasive study with positron emission tomography. Circulation 2001; 103: 1881-1886.

37 Gilbert EM, Abraham WT, Olsen S, et al. Comparative hemodynamic, left ventricular functional, and antiadrenergic effects of chronic treatment with metoprolol versus carvedilol in the failing heart. Circulation 1996; 94: $2817-2825$. 